\title{
A Internet das Coisas aplicada ao conceito de eficiência energética: uma análise quantitativo-qualitativa do estado da arte da literatura
}

\section{The Internet of Things applied to the energy efficiency concept: a quantitative-qualitative analysis of the state-of-the-art}

\author{
Arildo Antônio Sônego ${ }^{1}$, Roderval Marcelino ${ }^{1}$, Vilson Gruber $^{1}$ \\ ${ }^{1}$ Universidade Federal de Santa Catarina - UFSC, Florianópolis, SC, Brasil
}

Autor para correspondência/Mail to: Arildo Antônio Sônego (asonego@gmail.com)

Recebido/Submitted: 28 Jul. 2016; Aceito/Approved: 06 Set. 2016

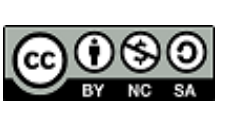
Copyright (C) 2016 Sônego, Marcelino \& Gruber. Todo o conteúdo da Revista (incluindo-se instruções, política editorial e modelos) está sob
uma licença Creative Commons Atribuição-NãoComercial-Compartilhalgual 3.0 Não Adaptada. Ao serem publicados por esta Revista, os arti-
gos são de livre uso em ambientes educacionais, de pesquisa e não comerciais, com atribuição de autoria obrigatória. Mais informações em
http://revistas.ufpr.br/atoz/about/submissions\#copyrightNotice.

\begin{abstract}
Resumo
Introdução: A Internet das Coisas corresponde a um novo paradigma, que imputa aos objetos a capacidade de disponibilizar informações a respeito de seu funcionamento e ao ambiente no qual tais objetos estão inseridos, caracterizando-se como abrangentes e diversificados os universos de prováveis aplicações a ela relacionadas. Em uma época em que se evidenciam ações e discussões dedicadas à sustentabilidade e ao meio ambiente, este artigo objetiva apurar o estado atual das pesquisas envolvendo a Internet das Coisas associado ao conceito de "eficiência energética".

Método: Tendo como referência a base de dados Scopus procedeu-se uma revisão sistemática da literatura, acompanhada de uma análise quantitativo-qualitativa. Foram aferidas informações no período compreendido entre os anos de 2001 a 2016, além da análise das cinquenta publicações com maiores citações.

Resultados: Constatou-se a existência de 895 documentos relacionados aos assuntos pesquisados. Contudo, poucos (foram identificados sete documentos) discorrem sobre a efetiva aplicação da Internet das Coisas à "eficiência energética".

Conclusão: Além de se determinar o estado da arte das pesquisas a nível mundial dos temas propostos, foi possível ratificar os conceitos a eles associados, bem como identificar as publicações mais relevantes, para que possam servir de referência e ponto de partida a outros trabalhos do gênero.
\end{abstract}

Palavras-chave: Internet das Coisas (IoT); Eficiência energética; Tecnologias de informação e comunicação (TIC)

\begin{abstract}
Introduction: The Internet of Things represents a new paradigm, which assign to objects the ability to provide information about its operations and the environment in which they live, characterized as a comprehensive and diverse universe and applications related to it. Nowadays, due to the recognition of actions and discussions devoted to sustainability and the environment, this article aims to assess the current state of research involving the Internet of Things, linked to the concept of "energy efficiency".

Method: Starting with reference to the database Scopus, it was conducted a systematic review of the literature, accompanied by a quantitative and qualitative analysis. The registers were collected using a 2001-2016 window, as well as it was made an analysis of fifty publications with higher citations. Results: It was found 895 documents related to the subjects studied. However, few (seven documents were identified) discussed the effective application of Internet of Things in "energy efficiency".

Conclusion: In addition to determining the state of the art research in the themes proposed, it was possible to ratify the concepts associated with them, as well as identify the most relevant publications which can provide a baseline for other investigations.
\end{abstract}

Keywords: Internet of Things (IoT); Energy efficiency; Information and communication technologies (ICT)

\section{INTRODUÇÃO}

Ao se analisar a adoção das Tecnologias de Informação e Comunicação (TIC) constata-se um crescimento considerável em recente período da sociedade moderna. Dispositivos como telefones celulares, tablets, smartphones e notebooks são frequentemente empregados em atividades cotidianas de profissionais das mais diferentes áreas, servindo também como fonte de diversão em momentos de relaxamento e lazer.

Conforme Barros (2011, p. 30), "[o]bserva-se, como em nenhum outro momento, o impacto da inclusão da tecnologia na vida das pessoas, atingindo a todos, de todas as maneiras, mesmo aqueles excluídos, vivendo em condições as mais adversas."e, de acordo com Nascimento (2011), a cada dia mais e mais pessoas, empresas, governos e outras organizações sociais tornam-se dependentes do uso contínuo de novas tecnologias, principalmente das Tecnologias de Informação e Comunicação. Entende-se, que as TIC tornaram-se elementos onipresentes ao ser humano.

A popularização do uso da Internet é considerada um dos motivadores deste processo. Na ótica de Pinho (2011, p. 98), "[p]arece que a Internet pode ser colocada como um marco civilizatório: a vida antes e depois da Internet, pois ela tem criado expectativas elevadas de mudanças, algumas até revolucionárias.". 
A Internet é provavelmente o maior sistema de engenharia já criado pela humanidade, com centenas de milhões de computadores conectados, enlaces de comunicação e comutadores, bilhões de usuários que se conectam por meio de laptops e tablets, e com uma série de dispositivos como sensores, webcams, consoles para jogos, quadros de imagens e até mesmo máquinas de lavar sendo conectadas (Kurose \& Ross, 2013).

Na concepção de Sato (2015), a rápida popularização da Internet - e seu acesso a partir de dispositivos móveis pode ser apontada como a parte mais visível das transformações pelas quais passa o cenário dos meios de comunicação, principalmente nas últimas décadas. Este cenário promissor apresenta-se favorável à manifestação de ideias, ao surgimento de conceitos e teorias, bem como à proliferação de novas tecnologias, entre elas, a Internet das Coisas (IoT - Internet of Things).

Em uma definição mais ampla, a Internet das Coisas relaciona-se à capacidade dos objetos, conectados em rede, disponibilizarem informações a respeito de seu funcionamento. Tal tecnologia, descreve Ferreira (2014), tem a finalidade de proporcionar inteligência para objetos, de modo a permitir seu controle e a notificação de alterações em seu estado.

Lacerda e Lima-Marques (2015, p. 159), afirmam que, "[i]nterligados em rede, os objetos são capazes de realizar ações de forma independente e gerar dados em quantidade e variedade exponenciais, como produto das interações".

Nesta conjuntura, entende-se que a dimensão, contexto e escopo de adoção de tais aplicações, apresentam-se como um dos desafios desta tecnologia. Segundo Wu, Talwar, K., Himayat, e Johnson (2011), não é possível representar de maneira exaustiva todas as alternativas de soluções atribuídas à Internet das Coisas, uma vez que o processo de compreensão do seu potencial ainda encontra-se em fase inicial, visto a variedade de aplicações que podem ser endereçadas em diversos segmentos do mercado. A Figura 1 contém exemplos de concentração proporcional por área (1-Insignificante, $5=$ Muito importante) de aplicações para a Internet das Coisas.

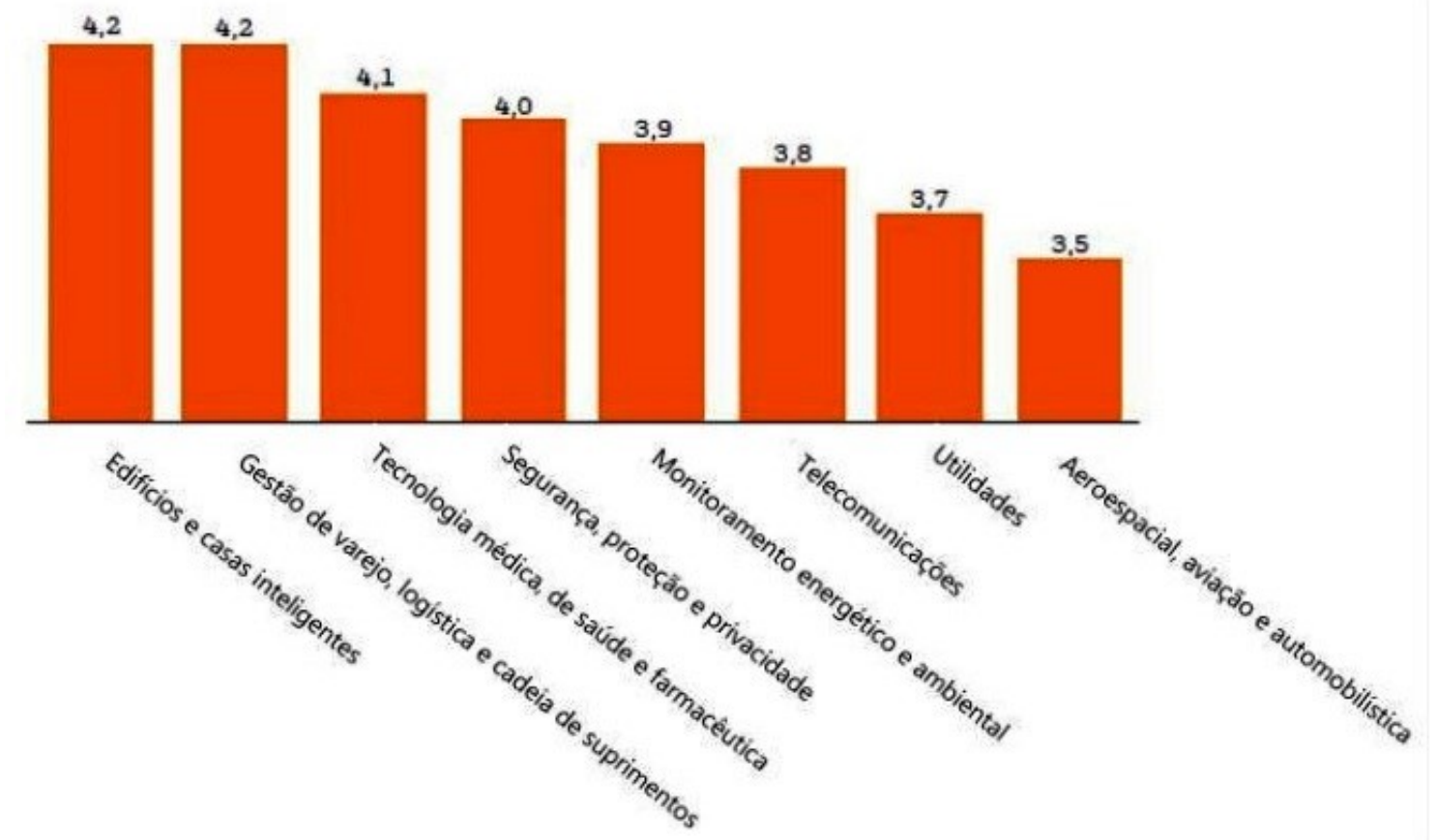

Figura 1. Áreas de concentração para a Internet das Coisas.

Fonte: Lacerda (2015, p. 72).

Uma das áreas elencadas anteriormente se relaciona à aplicação da Internet das Coisas no monitoramento energético e ambiental, sugerindo um uso adequado e consciente dos recursos naturais, contemplando a "eficiência energética".

O desenvolvimento econômico e os altos padrões de vida, destacam Hinrichs, Kleinbach, e Reis (2015), são processos complexos que necessitam de um abastecimento adequado e confiável de energia. Para Yu, Cecati, Dillon, e Simões (2011), a necessidade de minimizar ou mesmo equilibrar o impacto ao meio ambiente criado pelas atividades que fazem parte do estilo de vida do século XXI, promovendo o uso consciente dos recursos naturais, apresentam-se como um dos desafios da humanidade.

Desta maneira, de acordo com Wu et al. (2011), o processo de eficiência energética está condicionado à integração de novas tecnologias, como por exemplo, as TIC, sendo a Internet das Coisas considerada ideal para este ambiente. Assim, este artigo busca investigar a evolução das pesquisas no campo da Internet das Coisas objetivando compreender quais contribuições este paradigma pode oferecer ao conceito de "eficiência 
energética”.

\section{INTERNET DAS COISAS}

O conceito primordial associado à Internet das Coisas (IoT - Internet of Things) relaciona-se à capacidade que os objetos possuem de se comunicar, reportando informações acerca de seu estado e funcionamento. Segundo Serafim (2014), esta tecnologia consiste em interligar os objetos de uso cotidiano do ambiente real com a Internet, tornando-os então objetos inteligentes.

O termo Internet das Coisas, destaca Gogliano Sobrinho (2013), refere-se a um novo paradigma, que tem por premissa a integração entre objetos de uso cotidiano e a Internet. Contudo, sustenta o autor, para muitos esse conceito demonstra-se abstrato, e muitas vezes de difícil compreensão, no que tange à maneira de como se procede a essa integração.

Em 1999, o pesquisador do MIT (Massachusetts Institute of Technology) Kevin Ashton fez uso do termo Internet das Coisas pela primeira vez, em uma apresentação direcionada à empresa Procter \& Gamble (Serafim, 2014).

O significado do termo, explana Ferreira (2014), ampliou-se e passou a abranger a área de sensores e atuadores sem fio, de objetos conectáveis às redes que utilizam o protocolo TCP/IP (Transmission Control Protocol / Internet Protocol), assim como as tecnologias de semântica de dados, concebendo desta maneira, uma visão orientada às coisas, uma visão orientada à internet e uma visão orientada à semântica, conforme apresentado na Figura 2.

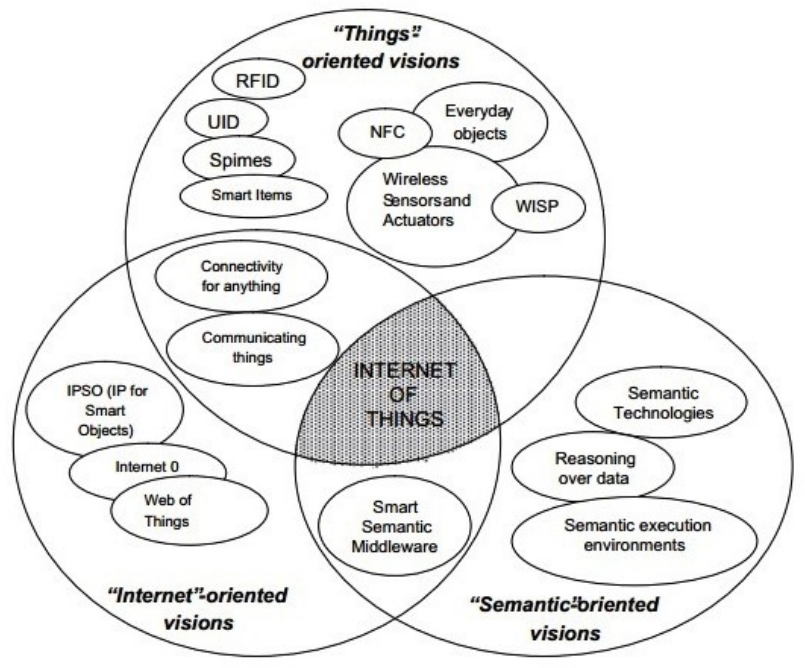

Figura 2. Diferentes visões da Internet das Coisas.

Fonte: Atzori, lera, e Morabito (2010, p. 2789).

Ao referirem-se às diferentes visões, Atzori et al. (2010) detalham que a visão orientada às coisas objetiva demonstrar propostas que assegurem o melhor aproveitamento dos recursos dos dispositivos e sua comunicação; a visão orientada à semântica foca na representação, armazenamento, pesquisa e organização da informação gerada, procurando soluções para a modelagem das descrições que permitam um tratamento adequado para os dados produzidos pelos objetos; enquanto que a visão orientada à internet tem o intuito de conceber modelos e técnicas destinadas a interoperabilidade dos dispositivos em rede.

A Internet das Coisas, preconiza Lacerda (2015), provê vários benefícios para a sociedade, concebendo efeitos significativos nas áreas de meio ambiente, saúde, comunicação, segurança, comodidade e urbanismo, uma vez que as aplicações são tantas quantas forem possíveis de se imaginar ao associar-se objetos com informações. Desta maneira, uma das aplicações sugeridas e investigadas neste artigo, diz respeito à eficiência energética.

\section{EFICIÊNCIA ENERGÉTICA}

De acordo com Roméro e Reis (2012), a energia está presente em todas as atividades humanas e, como não se encontra disponível de maneira direta na natureza - sendo obtida por meio de transformações de recursos naturais - seu consumo de forma adequada manifesta-se como um dos requisitos essenciais para a construção de um modelo de desenvolvimento sustentável. Burattini (2008) defende que a busca pela eficiência energética deve fazer parte consciente de todas as ações do ser humano moderno.

Para Pereira (2010), a utilização de energia tem sido intensiva e crescente desde a Revolução Industrial, uma vez que sua adoção é essencial para o funcionamento dos mais diversos setores e atividades da sociedade. Como 
exemplo, a autora cita a energia elétrica, responsável pelo funcionamento da maior parte dos equipamentos de edificações residenciais, comerciais e públicas. Na atualidade, complementa Oliveira (2015), emprega-se a energia elétrica para aquecer, refrescar, iluminar, preparar e conservar alimentos, gerenciar informações, entre outros.

Em termos de suprimento energético, sustenta Palz (2002), a eletricidade é o alicerce sobre o qual se encontra edificada a indústria eletrônica, a indústria dos aparelhos elétricos, uma considerável parte dos transportes públicos, a informática, bem como a indústria automobilística. Neste contexto, Burattini (2008, p. 47), reforça que, "[o] crescente desenvolvimento tecnológico envolvendo dispositivos que utilizam a eletricidade para seu funcionamento fez aumentar a demanda de energia elétrica".

Diversos são os meios de produzir energia elétrica, a partir de fontes renováveis ou não renováveis, conforme exemplificado na Figura 3, baseada na matriz elétrica brasileira, cada qual com suas vantagens e desvantagens econômicas e ambientais. Conforme Palz (2002), qualquer forma de energia pode ser transformada em eletricidade.

Uma fonte de energia é denominada renovável, destacam Goldemberg e Lucon (2012), quando as condições naturais possibilitam sua reposição em um curto espaço de tempo como, por exemplo: a energia solar, a energia eólica, a energia hidráulica e a biomassa (lenha, carvão vegetal, resíduos orgânicos). Em contrapartida, complementam os autores, as fontes não renováveis de energia são aquelas que a natureza não tem condições de repor em um horizonte de tempo compatível com seu consumo pelos seres humanos, como o carvão mineral, petróleo, gás natural e urânio.

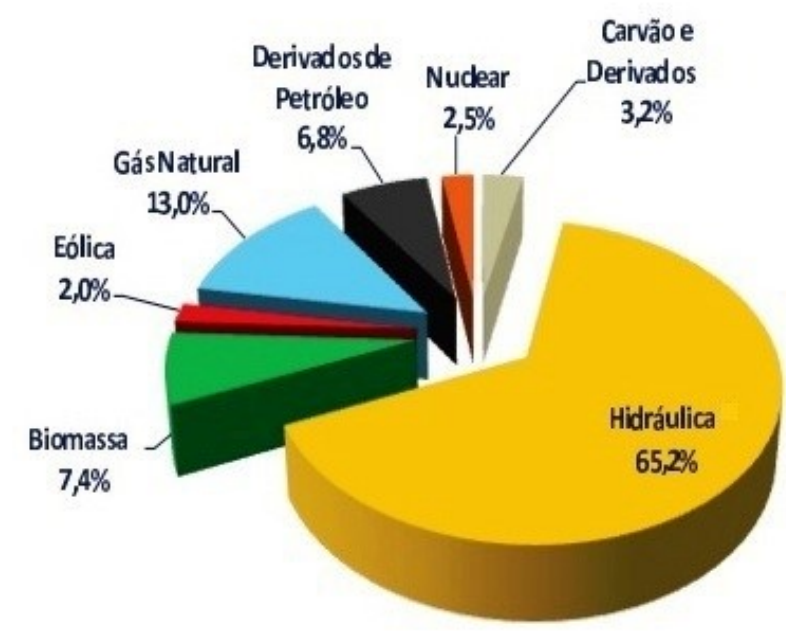

Figura 3. Matriz elétrica brasileira.

Fonte: Empresa de Pesquisa Energética [EPE] (2015, p. 35).

Ressalta-se a colaboração de Roméro e Reis (2012), que entendem que as fontes energéticas impactam com maior ou menor intensidade o meio ambiente, intensificando a necessidade de adoção de tecnologias menos poluentes sob qualquer aspecto. Neste cenário, Trsic e Fresqui (2012) ponderam que, o planeta Terra é um organismo vivo, sendo difícil introduzir novos corpos (tecnologias e afins) sem provocar algum tipo de reação.

Além do exposto, novas tecnologias, salientam Lamberts, Ghisi, Pereira, e Batista (2010) e Oliveira (2015), também contribuem para a Eficiência Energética. Dentre estas tecnologias, sugere-se a aplicação do paradigma de Internet das Coisas ao conceito de "eficiência energética”.

\section{METODOLOGIA}

Uma pesquisa consiste de uma investigação sistemática, que objetiva desenvolver teorias, estabelecer evidências e resolver problemas. É fundamental que o pesquisador esteja suficientemente informado do que foi pesquisado, como foi pesquisado, que resultados foram encontrados e o que ainda não foi pesquisado (Morandi \& Camargo, 2015).

Assim, para determinar a confiabilidade da pesquisa quanto aos seus resultados e análises, optou-se pela adoção da revisão sistemática da literatura e da análise quantitativo-qualitativa. Neste primeiro aspecto, Sampieri, Collado, e Lucio (2013, p. 76) ressaltam que a revisão sistemática, "[c]onsiste em detectar, consultar e obter a bibliografia e outros materiais úteis para os propósitos do estudo, dos quais extraímos e sintetizamos a informação relevante e necessária para o problema de pesquisa.". Os autores salientam que este procedimento 
deve ser seletivo, uma vez que todo ano, em diversas partes do mundo são publicados milhares de artigos em revistas acadêmicas, periódicos, livros e outros tipos de materiais nas diferentes áreas do conhecimento.

De acordo com Freire (2013), trata-se de um processo de levantamento de dados onde são exigidas revisões rigorosas de publicações acadêmicas em busca de indícios que possam levar à identificação de evidências a respeito de um tema de pesquisa ou mesmo um tópico em uma área desejada. Este método, ratificam Morandi e Camargo (2015), proporciona uma visão robusta e abrangente, permitindo que os pesquisadores se mantenham a par do que tem sido estudado em suas áreas de interesse.

Para a elaboração desta pesquisa, adotou-se a plataforma Scopus (www.scopus.com), base internacional responsável por publicações científicas de caráter multidisciplinar. Inicialmente realizou-se a análise estatística dos documentos, definindo-se como ponto de corte o período compreendido a partir de 2001 até 2016, uma vez que corresponde ao início do século XXI, englobando um universo de pesquisa de 15 anos, considerado como satisfatório para análises desta natureza. Para a realização da análise descritiva dos documentos, tendo como base este contexto, efetuou-se a escolha de 50 publicações utilizando como critério o número de citações que cada uma recebeu, em ordem decrescente. Por conseguinte, com base na leitura do resumo de cada obra selecionada, elencaram-se os documentos que apresentaram maior aderência ao assunto, excluindo-se aqueles que continham informações voltadas a universos diferentes do objeto de pesquisa proposto.

\section{RESULTADOS E DISCUSSÕES}

Como procedimento inicial, optou-se pela análise estatística dos documentos inserindo-se, na janela de busca da plataforma Scopus, o termo IoT, abreviatura para Internet of Things. A pesquisa reportou a ocorrência de 8.902 publicações, distribuídas segundo a Figura 4.

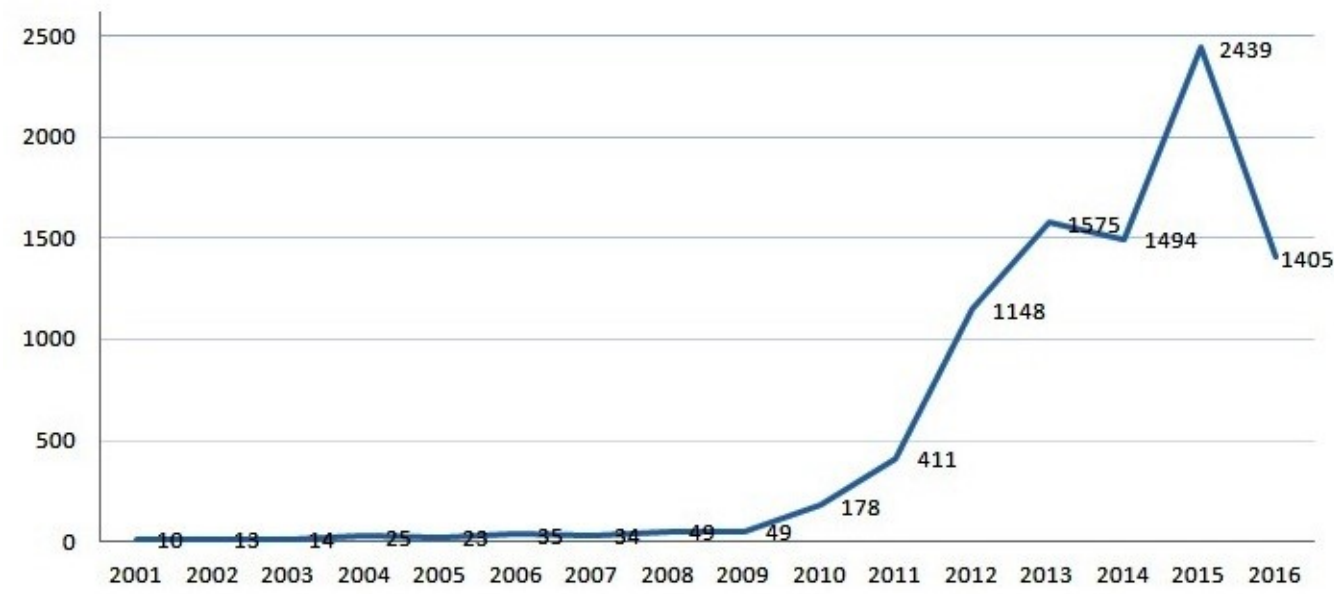

Figura 4. Evolução das pesquisas relacionadas à Internet das Coisas - Base Scopus (2001-junho/2016). Fonte: os autores.

Observa-se, apesar do termo ter sido utilizado pela primeira vez no ano de 1999, que apenas em 2010 iniciou-se o efetivo interesse pelo assunto, ocorrendo uma ligeira queda em 2014, sendo que o ápice ocorre em 2015. Por 2016 ser o ano em curso, ainda não há dados conclusivos sobre a pesquisa. Porém pela evolução do gráfico, acredita-se que esse número possa superar o de 2015.

Na seguinte pesquisa na base de dados Scopus, inseriram-se os termos energy efficiency, light efficiency e house efficiency, todos relacionados à “eficiência energética”. Ressalta-se que essa busca ainda não está associada à Internet das Coisas e sim aos assuntos e aplicações de uma maneira genérica. Como resultado, obteve-se a quantia de 346.761 documentos, conforme apresenta a Figura 5. Estes dados exibem valores bem mais expressivos quando comparados aos anteriores, relacionados à Internet das Coisas, indicando que a "eficiência energética” desperta considerável interesse por parte dos pesquisadores, pelo menos quantitativamente, devido, provavelmente, a ser um tema mais antigo. Todavia, conforme a Figura 5, em termos percentuais, as pesquisas voltadas diretamente à Internet das Coisas, apresentam evolução no crescimento a cada ano.

Nota-se também, um aumento constante no número de documentos publicados a cada ano e, tal qual a pesquisa anterior, o ano de 2015 contém uma maior quantidade quando comparada com os demais. Da mesma forma, como 2016 é o ano em curso, a quantidade de documentos ainda é reduzida, devido a não totalidade dos dados.

Posteriormente, visando filtrar as publicações associadas ao tema proposto, realizou-se a pesquisa associandose o termo Internet of Things aos termos energy efficiency, light efficiency e house efficiency. A pesquisa culminou com a exibição de 895 documentos, distribuídos de acordo com a Figura 6. Tal qual a Figura 4, relacionado aos 


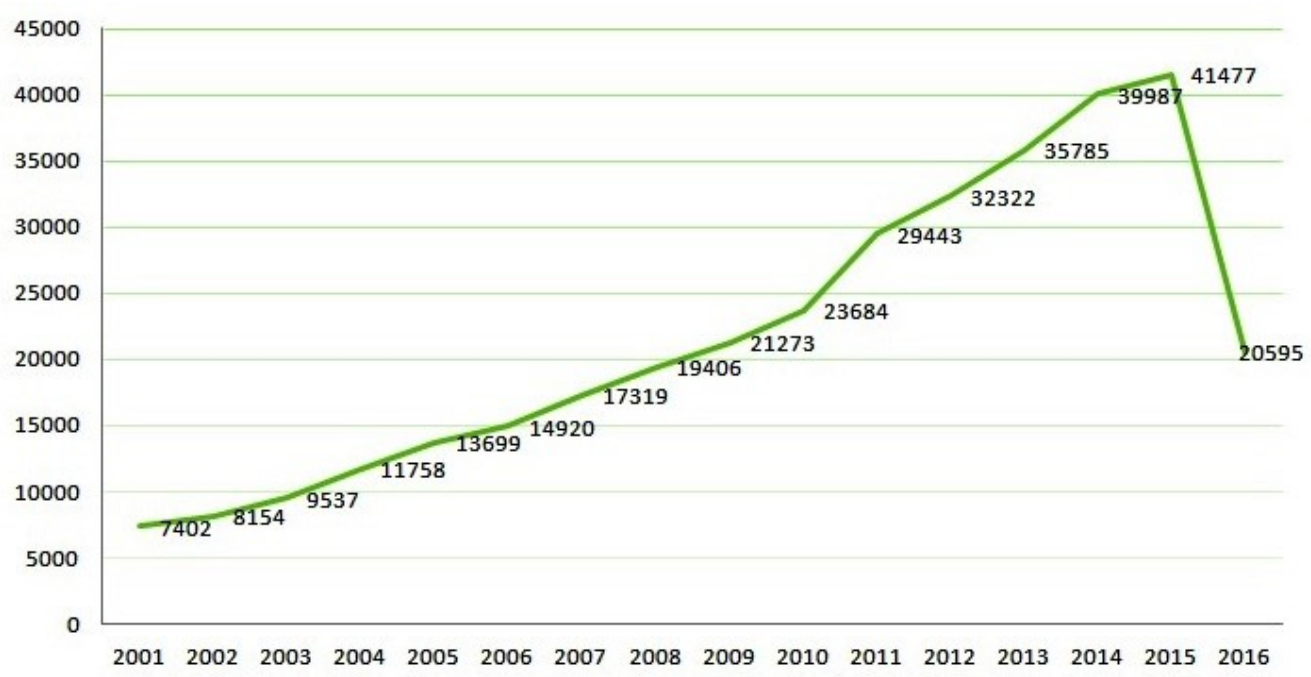

Figura 5. Evolução das pesquisas relativas à "eficiência energética" - Base Scopus (2001-junho/2016).

Fonte: os autores.

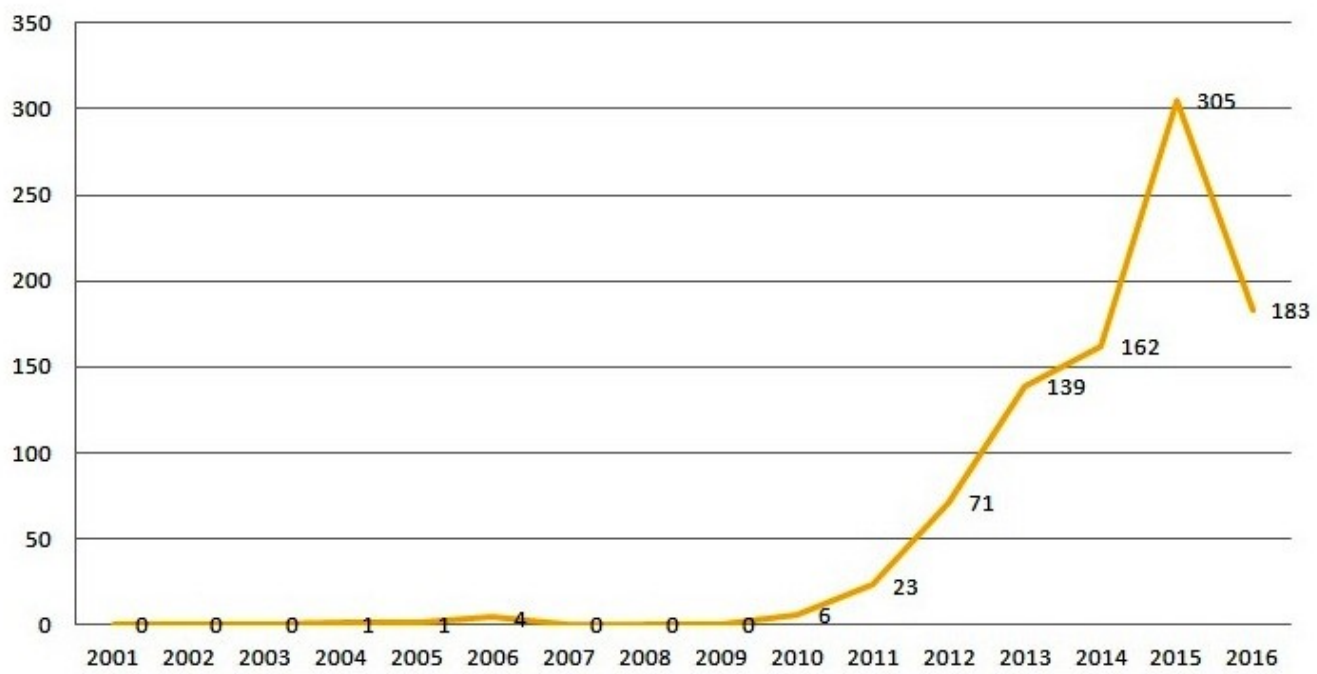

Figura 6. Publicações relacionadas à Internet das Coisas associada à "eficiência energética" - Base Scopus (2001-junho/2016). Fonte: os autores.

estudos a respeito da Internet das Coisas, observa-se uma evolução das pesquisas a partir do ano de 2010, sendo que em 2015 ocorrem as maiores quantidades.

A Figura 7 apresenta a distribuição de publicações por países, considerando os dez que mais contribuíram.

Constata-se uma predominância da China, com quase o dobro em relação aos Estados Unidos, segundo colocado. É interessante frisar que os dois, em conjunto, são responsáveis por quase metade (cerca de 43\%) de todas as publicações reportadas. Em relação ao Brasil, foram encontradas ao total, onze publicações, fazendo com que o país ocupe a vigésima posição.

A Figura 8 contém dados das publicações de acordo com seu tipo. Os papers, classificados como documentos publicados em conferências e seminários internacionais, são responsáveis pela maior quantidade de documentos, com 53,85\% das publicações, seguidos pelos artigos, documentos publicados em revistas e sites especializados, com $38,66 \%$. A soma dos dois tipos de documento totaliza $92,51 \%$, sendo que o restante é distribuído entre as demais formas.

Em se tratando de áreas de conhecimento, a Figura 9 apresenta os resultados obtidos.

A maioria dos documentos está associada às áreas de Computação, Engenharia e Matemática, sendo que apenas uma pequena parte demonstra-se atrelada especificamente à área energética. Atenta-se ao fato, que cada documento pode estar associado a mais de uma área específica.

O procedimento seguinte diz respeito à análise descritiva das publicações elencadas, conforme a quantidade de citações recebidas. Em termos de datas, o primeiro documento publicado intitula-se An improved concept for a Higher-Order Mode IOT e foi escrito por Bohlen e Wright (2004). No artigo, que não recebeu nenhuma citação, 


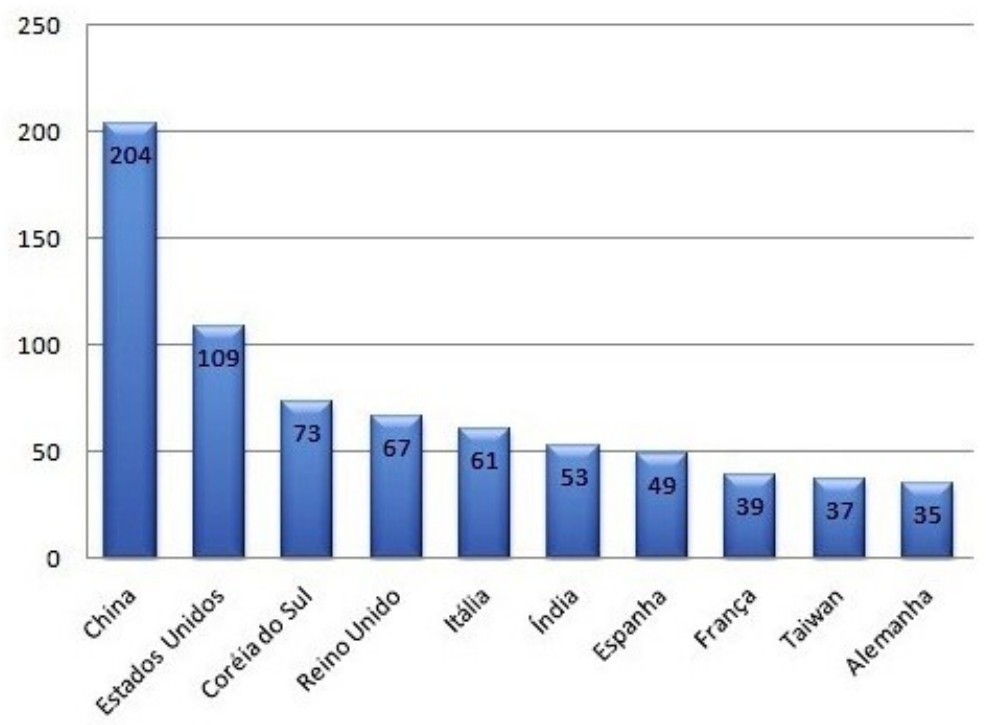

Figura 7. Publicações conforme o país de origem - Base Scopus (2001-junho/2016).

Fonte: os autores.

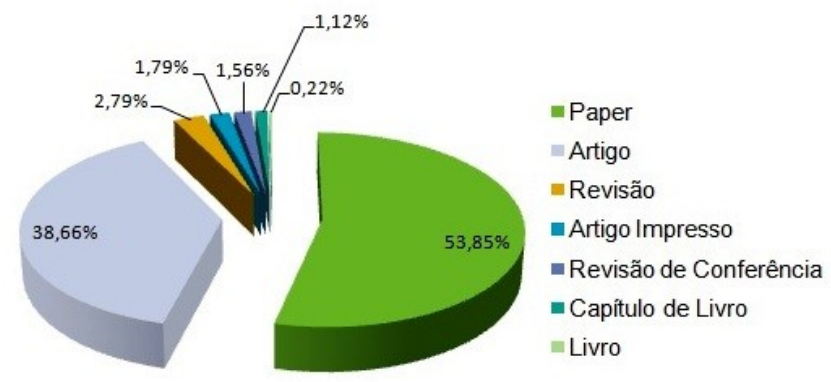

Figura 8. Publicações segundo o tipo - Base Scopus (2001-junho/2016).

Fonte: os autores.

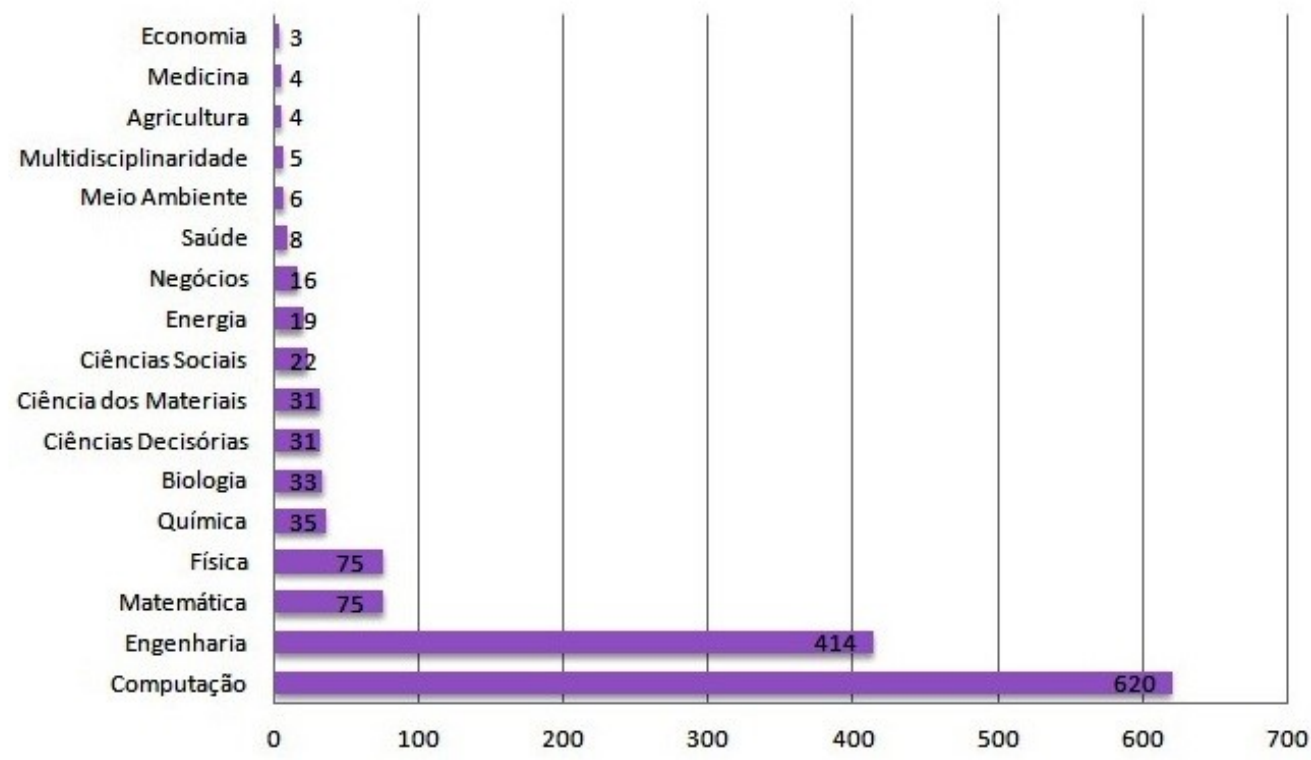

Figura 9. Publicações conforme a área de conhecimento - Base Scopus (2001-junho/2016).

Fonte: os autores.

os autores comentam sobre o desenvolvimento de fontes conversoras de voltagem, com eficiência energética na ordem de $62 \%$, a serem utilizadas em dispositivos eletrônicos.

O artigo Recommender systems survey, de Bobadilla, Ortega, Hernando, e Gutiérrez (2013), recebeu 259 citações, ficando em primeiro lugar neste quesito. $\mathrm{O}$ foco do documento concentra-se em sistemas de recomendação e 
sua afinidade com a Internet das Coisas, não fazendo menção à “eficiência energética”.

O trabalho de Sheng et al. (2013), denominado A survey on the ietf protocol suite for the internet of things: standards, challenges, and opportunities, apresenta aspectos relacionados à Internet das Coisas, enfatizando sua estrutura, principalmente os protocolos e tecnologias afins, como o IPv6, 6LoWPAN, 802.15.4 e ZigBee. Pontua-se também, sobre a necessidade da adoção de dispositivos que manifestem um reduzido consumo energético. $\mathrm{O}$ artigo apresenta uma quantidade de 101 citações a ele associadas. Nesta mesma linha de raciocínio, com considerações consonantes, está o artigo de Zeng, Guo, e Cheng (2011), intitulado The Web of Things: A survey, com 84 citações.

Aziz, Sekercioglu, Fitzpatrick, e Ivanovich (2013), no artigo A survey on distributed topology control techniques for extending the lifetime of battery powered wireless sensor networks, explanam sobre a utilização de energia em dispositivos associados à Internet das Coisas, principalmente em "redes de sensores sem fio". Segundo os autores, o uso eficiente deste recurso é fundamental para a sua operação e está condicionado à topologia da rede. Desta maneira, o artigo relata características, vantagens e desvantagens, demonstrando algoritmos que visam obter o máximo de eficiência da rede, subtraindo a menor quantidade possível de energia das baterias dos sensores. O documento foi citado 87 vezes.

O artigo How low energy is Bluetooth low energy? Comparative measurements with ZigBee/802.15.4, de Siekkinen, Hiienkari, Nurminen, e Nieminen (2012) é citado 47 vezes, faz um comparativo entre as tecnologias Bluetooth e ZigBee em relação ao consumo de bateria, enfatizando a tecnologia denominada BLE (Bluetooth Low Energy), considerada pelos autores, extremamente eficaz neste quesito. Como ponto negativo, existem restrições quanto à pilha de protocolos adotada pela BLE.

O trabalho de Hancke, de Carvalho e Silva, e Hancke Junior (2012), citado 46 vezes e denominado The role of advanced sensing in smart cities, apresenta aspectos de como a Internet das Coisas, juntamente com as redes de sensores sem fio podem contribuir para a sustentabilidade e o uso eficiente de recursos energéticos nas cidades, em áreas como: saúde, transportes, distribuição de água e energia, monitoramento ambiental e serviços públicos. Na percepção dos autores, a infraestrutura necessária para a concepção de um projeto deste porte, caracteriza-se por custos financeiros elevados e extrema complexidade, indicando a existência de consideráveis desafios a serem superados.

No artigo intitulado Internet of Things and BOM-based life cycle assessment of energy-saving and emission-reduction of products, escrito por Tao, Zuo, Xu, LV, e Zhang (2014), especificam-se procedimentos para reduzir o consumo de energia e minimizar a emissão de poluentes em processos industriais, utilizando a Internet das Coisas, propondo uma arquitetura de quatro níveis: acesso à percepção, dados, serviços e aplicação. Contudo, na ótica dos autores a pesquisa para produtos desta natureza está apenas começando, sendo que ainda existe um longo caminho a ser percorrido. Este artigo foi citado 15 vezes.

Huang, Meng, Gong, Liu, e Duan (2014), sugerem através do artigo A novel deployment scheme for Green Internet of Things, um ambiente voltado para a chamada Internet das Coisas Verde (Green IoT). Este artigo recebeu 14 citações e conforme os autores, o modelo proposto demonstra-se mais eficaz no consumo de energia quando comparado às tradicionais redes de sensores sem fio.

Em se tratando de documentos oriundos do Brasil, o artigo de Kamienski et al. (2015), denominado Context-aware energy efficiency management for smart buildings, discorre sobre a necessidade de aplicar conceitos relacionados à "eficiência energética" em prédios públicos, devido à estas edificações frequentemente serem desprovidas de mecanismos desta natureza. Para tal, os autores preconizam o uso da Internet das Coisas. O texto apresenta detalhes de como implementar esta filosofia e demonstra resultados obtidos através do desenvolvimento de um protótipo, baseado no microcontrolador Arduino, concebido para esta finalidade e contextualizado na sala de aula de uma universidade.

\section{CONCLUSÕES}

Neste artigo objetivou-se investigar a evolução das pesquisas relativas a associação da Internet das Coisas à Eficiência Energética, apresentando definições, aplicações e características inerentes a estes dois conceitos, através da adoção dos métodos de revisão sistemática da literatura e análise quantitativo-qualitativa.

A respeito do paradigma de Internet das Coisas (IoT), um ponto importante atenta-se justamente à definição e abrangência do termo. Contemplam-se incertezas quanto à maneira, intensidade e o contexto de sua concepção. Os autores pesquisados discordam em aspectos técnicos, e frequentemente defendem sua aplicação em circunstâncias pontuais, voltadas a cenários peculiares, demonstrando divergências em relação à essência do conceito.

Quanto aos aspectos inerentes à "eficiência energética", evidencia-se a dependência do ser humano pela eletricidade, sendo esta, fundamental para o desenvolvimento econômico, social e para o bem-estar. Demonstrase premente, a adoção de métodos de geração limpos, menos poluentes e agressivos ao meio ambiente, baseados 
em fontes renováveis. Tal qual estas medidas, a modificação dos hábitos de consumo da população e o incremento de novas tecnologias, se mostra relevante para alcançar resultados sustentáveis na utilização da energia.

Os dados obtidos pela revisão sistemática da literatura e pela análise bibliométrica, através da junção dos dois conceitos, sinalizam um extenso volume de publicações, indicando a relevância do tema. Em contrapartida, constata-se que os documentos discorrem acerca do uso eficiente de energia nos dispositivos, principalmente os sensores, associados à Internet das Coisas, apresentando pouca ênfase, em comparação à quantidade total de publicações existentes, na aplicação efetiva da tecnologia ao conceito de "eficiência energética", vislumbrandose desta maneira, um considerável universo investigativo a ser perscrutado. 


\section{REFERÊNCIAS}

Atzori, L., Iera, A., \& Morabito, G. (2010, Oct.). The Internet of Things: A survey. Computer Networks, 54(15), 2787-2805. doi: 10.1016/j.comnet.2010.05.010

Aziz, A. A., Sekercioglu, Y. A., Fitzpatrick, P., \& Ivanovich, M. (2013). A survey on distributed topology control techniques for extending the lifetime of battery powered wireless sensor networks. IEEE Communications Surveys \& Tutorials, 15(1), 121-144. doi: 10.1109/SURV.2012.031612.00124

Barros, T. H. C. (2011). Tecnologias da informação e comunicação (TICs) na educação: professores - mediadores mentores (Dissertaçao de Mestrado em Meios e Processos Audiovisuais, Universidade de São Paulo, Sao Paulo). Recuperado de http://www.teses.usp.br/teses/disponiveis/27/ 27161/tde-12032013-161611/pt-br.php

Bobadilla, J., Ortega, F., Hernando, A., \& Gutiérrez, A. (2013, July). Recommender systems survey. Knowledge-Based Systems, 46, 109-132. doi: 10.1016/j.knosys.2013.03.012

Bohlen, H. P., \& Wright, E. L. (2004). An improved concept for a higher-order mode IOT. In Fifth IEEE International Vacuum Electronics Conference (p. 311-). doi: 10.1109/IVELEC.2004.1316335

Burattini, M. P. T. C. (2008). Energia: uma abordagem multidisciplinar. São Paulo: Livraria da Física.

Empresa de Pesquisa Energética. (2015). Balanço Energético Nacional 2015 - Ano base 2014: Relatório Síntese. Rio de Janeiro: EPE. Recuperado de https://ben.epe.gov.br/downloads/S\%C3\%ADntese\% 20do\%20Relat\%C3\%B3rio\%20Final_2015_Web.pdf

Ferreira, H. G. C. (2014). Arquitetura de Middleware para Internet das Coisas (Dissertação de Mestrado em Engenharia Elétrica, Universidade de Brasília, Brasília). Recuperado de http://repositorio.unb.br/handle/10482/17251

Freire, P. S. (2013). Aumente a qualidade e a quantidade de suas publicações científicas: manual para elaboração de projetos e artigos científicos. Curitiba: Crv.

Gogliano Sobrinho, O. (2013). Serviço de resolução e descoberta de informações sobre objetos em sistemas baseados em RFID (Tese de Doutorado em Engenharia, Universidade de São Paulo, Sao Paulo). Recuperado de http://www.teses.usp.br/teses/disponiveis/3/3141/ tde-16102013-162918/pt-br.php

Goldemberg, J., \& Lucon, O. (2012). Energia, meio ambiente e desenvolvimento (3a. ed.). São Paulo: EDUSP.

Hancke, G. P., de Carvalho e Silva, B., \& Hancke Junior, G. P. (2012). The role of advanced sensing in smart cities. Sensors, 13(1), 393-425. doi: 10.3390/s130100393

Hinrichs, R. A., Kleinbach, M., \& Reis, L. B. (2015). Energia e meio ambiente (5a. ed.). São Paulo: Cengage Learning.

Huang, J., Meng, Y., Gong, X., Liu, Y., \& Duan, Q. (2014). A novel deployment scheme for Green Internet of Things. IEEE Internet of Things Journal, 1(2), 196-205. doi: 10.1109/jiot.2014.2301819

Kamienski, C., Borelli, F., Biondi, G., Rosa, W., Pinheiro, I., Zyrianoff, I., ... Pramudianto, F. (2015). Context-aware energy efficiency management for smart buildings. In 2015 IEEE 2nd World Forum on Internet of Things (WF-IoT) (p. 699704).

Kurose, J. F., \& Ross, K. W. (2013). Redes de computadores e a internet: uma abordagem top-down (6a. ed.). São Paulo: Pearson.
Lacerda, F. (2015). Arquitetura da informação pervasiva: projetos de ecossistemas de informação na internet das coisas (Tese de Doutorado em Ciência da Informação, Universidade de Brasília, Brasília). Recuperado de http://repositorio.unb .br/handle/10482/19646

Lacerda, F., \& Lima-Marques, M. (2015). Da necessidade de princípios de arquitetura da informação para a internet das coisas. Perspectivas em Ciência da Informação, 20(2), 158-171. doi: 10.1590/1981-5344/2356

Lamberts, R., Ghisi, E., Pereira, C. D., \& Batista, J. O. (2010). Casa eficiente: consumo e geração de energia. Florianópolis: UFSC LabEEE.

Morandi, M. I. W. M., \& Camargo, L. F. R. (2015). Revisão sistemática da literatura. In A. Dresch, D. P. Lacerda, \& J. A. V. Antunes Junior (Eds.), Design science research: método de pesquisa para avanço da ciência e tecnologia (p. 141-172). Porto Alegre: Bookman.

Nascimento, M. H. R. (2011). As tecnologias da informação e comunicação - TICs influenciam o desempenho acadêmico? (Dissertação de Mestrado em Economia, Universidade Católica de Brasília, Brasília). Recuperado de https://bdtd.ucb.br:8443/jspui/bitstream/123456789/454/ 1/Manoel\%20Henrique\%20Reis\%20Nascimento.pdf

Oliveira, I. P. (2015). Eficiência energética em sistemas de energia elétrica: um estudo de caso em uma indústria de lubrificantes (Dissertaçao de Mestrado em Eficiência Energética e Sustentabilidade, Universidade Federal de Mato Grosso do Sul, Campo Grande). Recuperado de http://repositorio.cbc.ufms.br:8080/jspui/bitstream/ 123456789/2602/1/IGORPETRIDEOLIVEIRA.pdf

Palz, W. (2002). Energia solar e fontes alternativas. Curitiba: Hemus.

Pereira, C. D. (2010). Introdução. In R. Lamberts, E. Ghisi, C. D. Pereira, \& J. O. Batista (Eds.), Casa eficiente:consumo e geração de energia (p. 17-21). Florianópolis: UFSC LabEEE.

Pinho, J. A. G. (2011). Sociedade da informação, capitalismo e sociedade civil: reflexões sobre política, internet e democracia na realidade brasileira. RAE: Revista de Administração de Empresas, 51(1), 98-106. doi: 10.1590/S003475902011000100009

Roméro, M. A., \& Reis, L. B. (2012). Eficiência energética em edifícios. Barueri: Manole.

Sampieri, R. H., Collado, C. F., \& Lucio, M. P. B. (2013). Metodologia de pesquisa (5a. ed.). Porto Alegre: Penso.

Sato, S. K. (2015). Mobilidade, comunicação e consumo: expressões da telefonia celular em Angola, Brasil e Portugal (Tese de Doutorado em Comunicação, Universidade de São Paulo, São Paulo). Recuperado de http://www.teses.usp.br/teses/ disponiveis/27/27153/tde-01062015-163806/pt-br.php

Serafim, E. (2014). Uma estrutura de rede baseada em tecnologia IoT para atendimento médico a pacientes remotos (Dissertação de Mestrado em Ciência da Computação, Faculdade Campo Limpo Paulista, Campo Limpo Paulista). Recuperado de http://www.cc.faccamp.br/Dissertacoes/Edivaldo 2014.pdf

Sheng, Z., Yang, S., Yu, Y., Vasilakos, A. V., Mccann, J. A., \& Leung, K. K. (2013). A survey on the ietf protocol suite for the internet of things: standards, challenges, and opportunities. IEEE Wireless Communications, 20(6), 91-98. doi: 10.1109/mwc.2013.6704479

Siekkinen, M., Hiienkari, M., Nurminen, J. K., \& Nieminen, J. (2012). How low energy is bluetooth low energy? comparative 
measurements with ZigBee/802.15.4. In 2012 IEEE Wireless Communications and Networking Conference Workshops ( $\mathrm{p}$. 232-237). doi: 10.1109/wcncw.2012.6215496

Tao, F., Zuo, Y., Xu, L., LV, L., \& Zhang, L. (2014). Internet of Things and BOM-based life cycle assessment of energy-saving and emission-reduction of products. IEEE Transactions on Industrial Informatics, 10(2), 1252-1261. doi: 10.1109/tii.2014.2306771

Trsic, M., \& Fresqui, M. C. (2012). Curso de química para engenharia. Barueri: Manole.

Wu, G., Talwar, S., K., J., Himayat, N., \& Johnson, K. D. (2011). M2M: from mobile to embedded internet. IEEE Industrial Electronics Magazine, 49(4), 36-43. doi: 10.1109/mcom.2011.5741144

Yu, X., Cecati, C., Dillon, T., \& Simões, M. G. (2011). The new frontier of smart grids. IEEE Industrial Electronics Magazine, 5(3), 49-63. doi: 10.1109/mie.2011.942176

Zeng, D., Guo, S., \& Cheng, Z. (2011). The Web of Things: A survey. Journal of Communications, 6, 424-438. doi: 10.4304/jcm.6.6.424-438

Como citar este artigo (APA):

Sônego, A. A., Marcelino, R. \& Gruber, V. (2016). A Internet das Coisas aplicada ao conceito de eficiência energética: uma análise quantitativo-qualitativa do estado da arte da literatura. AtoZ: novas práticas em informação e conhecimento, 5(2), 80 - 90. Recuperado de: http://dx.doi.org/10.5380/atoz.v5i2.47860 